Revue d'histoire de l'Amérique française

REVUE D.HISTOIRE DE L'AMÉRIQUE FRANÇAISE

\title{
Qui était « Pierre-André », le premier critique de notre premier roman?
}

\section{Yves Garon}

Volume 20, numéro 4, mars 1967

URI : https://id.erudit.org/iderudit/302615ar

DOI : https://doi.org/10.7202/302615ar

Aller au sommaire du numéro

Éditeur(s)

Institut d'histoire de l'Amérique française

ISSN

0035-2357 (imprimé)

1492-1383 (numérique)

Découvrir la revue

Citer cet article

Garon, Y. (1967). Qui était " Pierre-André », le premier critique de notre premier roman? Revue d'histoire de l'Amérique française, 20(4), 566-571. https://doi.org/10.7202/302615ar d'utilisation que vous pouvez consulter en ligne.

https://apropos.erudit.org/fr/usagers/politique-dutilisation/ 


\section{QUI ETTAIT "PIERRE-ANDRE", LE PREMIER CRITIQUE DE NOTRE PREMIER ROMAN?}

A la mi-septembre 1837, paraissait L'Influence d'un Livre de Philippe-Aubert de Gaspé (fils), le premier roman écrit par un Canadien français. Dans le Populaire du 25 septembre de cette même année, un entrefilet intitulé "De l'influence d'un livre" s'en prenait à la préface un peu fracassante dont De Gaspé avait fait précéder son roman. Ce court article n'était pas signé, mais il faut l'attribuer, selon Monsieur Luc Lacourcière (voir l'article très érudit qu'il consacre à "Philippe-Aubert de Gaspé (fils), dans: Livres et Auteurs canadiens 1964, Montréal, Éditions Jumonville, p. 150-157), à l'Éditeur en chef du Populaire, Hyacinthe Leblanc de Marconnay.

Les remarques de ce journaliste français ne sont pas sans intérêt, mais elles restent brèves, voire hâtives, et nous croyons que l'on peut tenir pour la première critique de notre premier roman celle qui parut quelques jours plus tard, le 11 octobre, dans ce même Populaire d'ailleurs. Elle était signée du pseudonyme jusqu'ici énigmatique de "Pierre-André". De Gaspé défendit son œuvre, et il y eut une nouvelle intervention de PierreAndré. La critique et les répliques qu'elle suscita sont fort intéressantes; Luc Lacourcière les analyse dans l'article mentionné plus haut, et il ajoute: "Avant de clore cette petite bataille romantique autour de notre premier roman, il reste à nous demander qui était Pierre-André. D'après sa collaboration régulière au Populaire, on peut déduire que c'était le pseudonyme d'un tout jeune homme qui disait avoir seize ans et demeurait aux TroisRivières. Audet et Malchelosse l'identifient (Pseudonymes canadiens) comme étant Guillaume Barthe. Mais cela nous semble insuffisamment prouvé. D'abord l'âge ne concorde pas. Barthe 
est né en 1816. Condisciple du jeune De Gaspé au collège de Nicolet, il était resté en relations avec lui et semblait professer à son endroit une admiration certaine. Il dira dans ses Mémoires:

Philippe Aubert de Gaspé, l'auteur de ce livre charmant L'Influence d'un livre, qui fit dans le temps, sa sensation... venait de fois à autres passer quelques jours à Trois-Rivières, chez M. Vallières, ce qui était une bonne fortune pour moi en ce qu'il me procurait des récréations nouvelles...

Les Mémoires de Barthe sont de 1885. Le temps aurait-il pu atténuer ses impressions premières ? Voici un autre témoignage, cette fois contemporain de la querelle. Dans une épître intitulée Aux littérateurs canadiens, il célèbre à l'occasion du nouvel an, les jeunes écrivains:

Deux brillants lauréats ont surchargé leurs fronts

Des palmes orgueilleux (sic) des poétiques monts:

Le jeune Pierre-André, l'aimable Solitaire,

Deux chantres orgueilleux, qu'en ce coin de la terre,

Les reines du Parnasse honorent de leurs dons

Le nom de De Gaspé, par dessus mille noms,

Surgit, tout glorieux, exalté par les muses:

Il grandira toujours pour la gloire des muses !

Ces vers "glorieux" autant que gauches et fautifs qui exaltent De Gaspé ont paru dans le Populaire du 17 janvier 1838, sous la signature de Barthe. Mais "l'aimable Solitaire", ainsi vanté, serait Barthe lui-même; et il n'est pas impossible, malgré tout, que Barthe ait joué double jeu et que Pierre-André soit un autre de ses dédoublements." (Lacourcière, op. cit., 155-156).

Nous avions tenté de notre côté, il y a deux ans, de découvrir quel personnage se cachait sous le pseudonyme de PierreAndré, sans y parvenir. Mais, cet été, des recherches nous ont ramené au Populaire et nous avons pu, après quelques tâtonnements, établir d'une manière irréfutable l'identité de PierreAndré: il s'agit de Romuald Cherrier.

Et voici comment nous avons pu faire cette identification. La signature de Pierre-André n'apparaît pas seulement à la fin de sa critique de L'Influence d'un Livre; nous la retrouvons 
au bas de quinze poèmes (et d'autres compositions) publiés dans ce même Populaire entre le 11 septembre 1837 et le 24 janvier 1838. Or cinq de ces quinze pièces figurent dans le Répertoire national compilé par James Huston, mais signées cette fois du nom véritable de leur auteur, à savoir Romuald Cherrier. Ces pièces sont: "A mon amie", dans le Populaire, 11 septembre 1837, (Répertoire national), 2e édition, 1893, Vol. II : 21) ; "Élégie sur la mort de Hugues Heney, fils", dans le Populaire, 18 septembre 1837, (Répertoire national, Vol. II: 135-136); "A mon frère", (traduction libre de l'anglais), dans le Populaire, 20 octobre 1837, (Répertoire national, Vol. II : 25) ; "A ma sœur, le bouquet de naissance", dans le Populaire, 15 janvier 1838, (Répertoire national, Vol. II : 83-85) ; enfin, "Élégie. Des pleurs à la mémoire d'Antonin", dans le Populaire, 24 janvier 1838, (Répertoire national, Vol. II : 76).

Cette première preuve est à elle seule suffisante, mais on peut y joindre de nombreuses autres confirmations. En voici quelques-unes. Pierre-André, qui apparemment tient à ce qu'on sache qu'il est écrivain précoce, indique ou fait dire à son éditeur qu'il a composé tel poème à treize ans et huit mois (Le Populaire, 11 décembre 1837), tel autre à quatorze ans (Le Populaire, 18 septembre 1837). En janvier 1838, il communique au journal un poème, "A ma sœur, le bouquet de naissance", et réclame l'indulgence des lecteurs en déclarant que cette pièce, il l'a adressée à sa sœur quelque deux ans plus tôt, alors qu'il n'avait pas encore atteint sa quinzième année. Pierre-André était donc un jeune homme de seize ans en 1837. Or, Romuald Cherrier est né le 6 mars 1821, à Montréal (Mémoires de la Société généalogique canadienne-française, Vol. II, no 3, janvier 1947: 152), et fut baptisé André-Romuald.

Une autre confirmation. Le Populaire du 18 septembre 1837 publie "Élégie sur la mort de Hugues Heney, fils" de Pierre-André, et l'éditeur ajoute au poème la note suivante: "Nous avons cru devoir reproduire ces vers, quoiqu'ils aient déjà été insérés dans L'Ami du peuple du 27 août $1836 \ldots$.." Or, ce poème n'est pas, dans L'Ami du peuple, signé Pierre-André, 
mais "A.R.C.", qui sont, on le voit, les initiales d'André-Romuald Cherrier.

Autre preuve encore. Pierre-André a adressé quelques poèmes à sa "scur", et l'un d'entre eux intitulé "Un rêve d'or" porte une dédicace précise: "A Odile" (Le Populaire, 22 septembre 1837), et cette sœur, il la donne comme un peu plus âgée que lui (Le Populaire, 15 janvier 1838, la note qui suit le poème en particulier). Or, Romuald Cherrier avait une sœur appelée Josephte-Odile, née le 28 novembre 1819, donc plus âgée que Romuald de 15 mois environ. Cette sœur de Romuald envoyait d'ailleurs elle-même des compositions au Populaire et elle signait "Anaïs". Nous avons pu l'identifier grâce encore une fois au Répertoire national. Celui-ci reproduit en effet (vol. II: 137-141) un morceau intitulé "Une scène à SaintDomingue", et signé Mlle Odile Cherrier. Or, ce même récit avait paru dans le Populaire du 17 janvier 1838, sous le titre: "Horrible tragédie (Traduction libre de l'anglais), Une scène à Saint-Domingue", et il était signé Anaïs. D'autres envois d'Anaïs apportent de nouvelles confirmations. Ainsi elle fait paraître "Une promenade avec André, dans le cimetière" (Le Populaire, 2 février 1838), "L'Amitié" (A mon frère), (Le Populaire, 12 février 1838 ).

Une dernière confirmation touchant l'identité de PierreAndré. On sait qu'en même temps que Romuald et Odile Cherrier, Guillaume Barthe écrivait dans le Populaire. Il fut même l'auteur d'une petite mystification, puisqu'au début il signait Marie-Louise, ce qui lui valut les hommages poétiques de PierreAndré et de quelques autres (le Populaire, 20 septembre, 4 et 18 octobre 1837, etc.). Il devait mettre fin à ce petit amusement après quelque temps en permettant à l'éditeur de révéler son identité (le Populaire, 6 novembre 1837). Ainsi, pendant plusieurs mois, trois pseudonymes étaient revenus sans cesse dans le Populaire: Marie-Louise, Pierre-André et Anaïs. Or, parmi les huit enfants de Romuald Cherrier, nous voyons une fille qu'il fait appeler Louise-Anaïs-Odile, une autre qu'il nomme MarieLouise-Eveline, un de ses fils portera le prénom d'André. 
(Mémoires de la Société généalogique..., loc. cit.). Romuald Cherrier gardait, de son aventure littéraire dans le Populaire, une certaine nostalgie et avait pour sa sœur un amour profond, d'où les prénoms de ses enfants: Marie-Louise, Anaïs, Odile...

Nous aurions pu relever de nombreux autres détails qui montrent à l'évidence que Pierre-André est Romuald Cherrier et Anaïs, sa sœur Odile, mais cela est superflu: l'identité de ces deux écrivains étant bien établie par ce qui précède.

Romuald était le fils d'un "marchand-pelletier, manchonnier" de Montréal et le benjamin de la famille qui comptait quatre enfants: l'aîné, Georges-Hippolyte (1813-1903), fut, en 1853, l'éditeur de Charles Guérin de P. J. O. Chauveau et de la revue $L a$ Ruche littéraire que dirigea $\mathrm{H}$. E. Chevalier; le second fils, Trefflé (1818- ), fut agent des terres de la province, à Québec, de 1860 à 1890 ; leur sœur Odile, qui écrivit dans le Populaire sous le pseudonyme d'Anaïs, devait épouser Prudent Vallée qui fut président de la Banque Nationale de Québec. Leur père mourut à quarante-deux ans, en 1828. Romuald n'avait alors que sept ans, sa sœur neuf ans et ses frères dix et quinze ans. Romuald, comme ses deux frères, fit ses études au Collège de Montréal (1832-1840); il fut admis au Barreau en 1842. Il épousa Henriette Parthenais le 7 octobre 1844 à Joliette. Après avoir exercé sa profession à Montréal pendant une dizaine d'années, il alla s'établir à Joliette en 1853. Le surmenage devait le conduire à une mort prématurée, en 1863. (Mémoires de la Société généalogique..., loc. cit.).

Le Répertoire national a conservé sept poèmes de Romuald Cherrier (Vol. II : 21, 25, 74, 76, 83, 135, 226). Cinq d'entre eux, comme nous le disions plus haut, avaient paru dans le Populaire avec quelques brefs essais. Il serait aussi, sous le pseudonyme de "Un étudiant en droit", l'auteur de Procès de Joseph $N$. Cardinal et autres, auquel on a joint la requête argumentative en faveur des prisonniers, et plusieurs autres documents précieux, etc., Montréal, John Lovell, 1839, 144 p. (Audet et Malchelosse, Pseudonymes canadiens, Montréal, Ducharme, 1936, 59). 
Cette question de l'identité de Pierre-André était peut-être d'une importance assez mince, mais ce sont les petits ruisseaux qui font la rivière et les détails, l'Histoire. Il y aurait intérêt à étudier davantage la vie et l'œuvre de Romuald Cherrier, et, plus encore, le rôle littéraire joué par le Populaire. Cela sera peut-être plus séduisant maintenant que certains pseudonymes ne font plus écran.

YVES GARON, a.a.

Département d'études canadiennes, Université Laval.

Avec ce présent numéro prend fin la 20e année de la REVUE D'HISTOIRE DE L'AMÉRIQUE FRANÇAISE et la dernière livraison de l'année (juin 1966-1967). Prière de renouveler votre abonnement, au prix de $\$ 6.00$ (abonnement de soutien : $\$ 10.00$ ), avec le billet vert inclus.

L'index de la deuxième décennie paraîtra fin avril ou début de mai. Il nous reste encore des index de la première décennie que nous pouvons aussi vendre au même prix : $\$ 6.00$ l'exemplaire. 